\title{
SCIENTIFIC Poster Presentation AbStraCtS
}

method for classification of AD and CN was better than using one classifier and comparable with stateof-the-art methods.

Keywords: Ensemble Machine Learning; Alzheimer's Disease; Discrete Wavelet Transform; Principal Component Analysis; Statistics Features; Magnetic Resonance Imaging

\section{• 10.5812/IRANJRADIOL.99158}

\section{A Proposal to Approach Cloud-Based Enterprise Imaging for Medical Universities in Iran Using Existing DICOM Infrastructure}

\author{
Masood Raeesi ${ }^{1,}$ *; Mansoor Fatehi' ${ }^{2}$; Mahdi Shamsi ${ }^{3}$; Navid \\ Towfighirad $^{3}$ \\ ${ }^{1}$ Healthcare IT (HIT) Department, Taimaz Co., Tehran, Iran \\ ${ }^{2}$ Virtual University of Medical Sciences, Tehran, Iran \\ ${ }^{3}$ Radiology Department, Laleh Hospital, Tehran, Iran \\ ${ }^{*}$ Corresponding author: Healthcare IT (HIT) Department, Taimaz Co., \\ Tehran,Iran.Email:masoodraeesi@yahoo.com
}

\begin{abstract}
Background:Exchange and share of different medical images as one of the critical parts of patients' medical records between different departments of the same hospital or even different hospitals have always been in demand in recent years. Medical universities in Iran include affiliated hospitals and medical centers. Most of those hospitals were equipped with picture archiving and communication systems (PACS) from different vendors in the past years. The main challenges concerning the functionality of current PACS-based workflow with those vendors are the lack of functionality to capture, store, and view DICOM and non-DICOM images from other departments other than radiology, as well as the lack of communication and exchange of medical imaging among hospitals since each vendor has its own protocol of communication.

Objective: Some advantages of this demand include allowing healthcare providers for on-demand access to medical imaging acquired at other affiliated hospitals using authentication, reducing unnecessary repeated imaging exams and unnecessary exposure to radiation, better managing the information technology resources in the Cloud environment, satisfying patients and healthcare providers, and providing timely access to patient medical imaging history.
\end{abstract}

Methods: In this study, we proposed a private Cloud- based enterprise imaging solution for each medical university based on the existing DICOM infrastructure, which was supported by commonly accepted standards of all vendors. In each hospital, non-DICOM medical images were converted via a "Dicomizer" to DICOM and beside native DICOM images were sent via a "Router" module to the Central Archiving solution located in private Cloud of the Medical University. By utilizing the Router module, it was possible to transfer images even in narrow bandwidth lines via lossless/lossy compression under TLS/SSL protocol, making necessary DICOM coercions and adding DICOM tags and facilitating the possibility of having some other useful features. A zero-footprint viewer was considered in the Cloud environment for the purpose of anytime/anywhere viewing of patient studies.

Results: The primary test of the Router module even through the internet network was encouraging. It seemed this module could work much more effective through the intranet of medical universities and the affiliated hospitals.

Discussion: Besides transferring images by Router, this module could add or coerce some necessary DICOM tags, facilitating the categorization of studies, automatic pseudonymization of patient data, and support of all DICOM store classes including nonimage classes like structured reports.

Conclusion: The proposed method of archiving Enterprise Imaging in private cloud using the existing DICOM infrastructure seems feasible, cost-effective, and convenient since it does not affect the current workflow of archiving of medical images in affiliated hospitals of medical universities in Iran and there is no concern about different communication protocols. By full implementation of this proposal, each healthcare provider would have on-demand access to patient studies upon authentication without further need to store departmental silos of data. Keywords: DICOM; PACS; Enterprise Imaging; Cloud Computing

\section{• 10.5812/IRANJRADIOL.99159}

\section{Automatic Detection and Classification of White Blood Cells in Blood Smear Images Using Convolutional Neural Network}

\footnotetext{
Ramin Nateghi ${ }^{1,}$ *; Mansoor Fatehi ${ }^{2}$; Ali Sadeghitabar ${ }^{3}$; Romana Khosravi ${ }^{3}$; Fattane Pourakpour ${ }^{2}$

${ }^{1}$ Department of Electrical and Electronic Engineering, Shiraz University of Technology, Shiraz, Iran
} 